Quim. Nova, Vol. 31, No. 5, 1048-1052, 2008

\title{
PREPARAÇÃo E CARACTERIZAÇÃo DE CARVÃo ATIVAdO PRODUZIDO A PARTIR DE RESÍDUOS DO BENEFICIAMENTO DO CAFÉ
}

\author{
Sarah Silva Brum, Maria Lucia Bianchi*, Vanésia Liane da Silva, Maraísa Gonçalves, Mário César Guerreiro e Luiz \\ Carlos Alves de Oliveira \\ Departamento de Química, Universidade Federal de Lavras, CP 3037, 37200-000 Lavras - MG, Brasil
}

Recebido em 23/3/07; aceito em 14/11/07; publicado na web em 24/7/08

\begin{abstract}
PREPARATION AND CHARACTERIZATION OF ACTIVATED CARBON PRODUCED FROM COFFEE WASTE. Coffee fruit processing is one of the most polluting activities in agriculture due to the large amount of waste generated in the process. In this work, coffee parchment was employed as precursor for the production of carbons activated with $\mathrm{ZnCl}_{2}(\mathrm{CAP})$. The material was characterized using $\mathrm{N}_{2}$ adsorption/desorption at $77 \mathrm{~K}$, Fourier transform infrared spectroscopy (FTIR) and scanning electron microscopy (SEM). The material showed a surface area of $521.6 \mathrm{~m}^{2} \mathrm{~g}^{-1}$ and microporous structure. CAP was applied as adsorbent for the removal of methylene blue dye in aqueous medium. The adsorption capacity was found to be about $188.7 \mathrm{mg} \mathrm{g}^{-1}$.
\end{abstract}

Keywords: activated carbon; coffee waste; adsorption.

\section{INTRODUÇÃO}

O Brasil é um dos maiores produtores mundiais de café, com safra 2007/2008 estimada em 43 milhões de sacas de $60 \mathrm{~kg} .{ }^{1}$ Durante o processo de industrialização dos grãos de café a quantidade de resíduo gerada é igual à quantidade de café beneficiado produzido, ou seja, para se obter $1 \mathrm{~kg}$ de grãos beneficiados, são necessários $2 \mathrm{~kg}$ de café em coco. ${ }^{2}$ Assim, somente o beneficiamento do café irá gerar cerca de 2,6 milhões de toneladas de resíduos. Esses resíduos (material lignocelulósico), se dispostos inadequadamente, podem causar a poluição de solos e águas.

Uma alternativa para evitar o descarte desses resíduos seria sua utilização na preparação ou produção de material de maior valor agregado, transformando o lixo em matéria prima.

O pergaminho, conhecido como "casquinha" por ser uma película anatômica que envolve o grão, é parte do resíduo gerado pelo beneficiamento do café, obtido quando a despolpa é feita por via úmida. Esse material se caracteriza quimicamente pelo seu alto teor de fibra bruta (celulose, lignina e hemicelulose) e baixos teores de extrativos e cinzas. ${ }^{3}$

Em anos recentes, vários estudos relataram a produção de carvões ativados (CA) a partir de resíduos tais como sementes de frutas, ${ }^{4}$ bagaço de cana-de-açúcar, ${ }^{5}$ restos de couro, ${ }^{6}$ pneus ${ }^{7}$ etc. O carvão ativado (CA) é um material carbonáceo e poroso preparado pela carbonização e ativação de substâncias orgânicas, principalmente de origem vegetal. ${ }^{8}$ São utilizados extensamente para a adsorção de poluentes em fases gasosas e líquidas, como suporte para catalisadores, na purificação de vários compostos, no tratamento de efluentes, etc. A capacidade de adsorção do CA depende da natureza do precursor, do tamanho das partículas (grânulos ou pó) e do processo de produção escolhido. A qualidade dos CAs é avaliada em termos de suas propriedades físicas de adsorção e da área superficial. ${ }^{8-10}$

O presente trabalho teve como objetivo o uso de um resíduo do beneficiamento do café (pergaminho) na preparação de carvão ativado (material de maior valor econômico). O material produzido foi caracterizado quanto a sua área superficial específica, parâmetros

*e-mail: bianchi@ufla.br termodinâmicos e capacidade de adsorção do corante azul de metileno (AM).

\section{PARTE EXPERIMENTAL}

\section{Amostragem}

O pergaminho foi obtido do beneficiamento do café por despolpa úmida na fazenda da EPAMIG em Machado, MG, em julho/agosto de 2005. O material utilizado foi seco e moído, resultando numa granulometria entre 250 a $420 \mu \mathrm{m}$.

\section{Caracterização do pergaminho}

Os teores de carbono, hidrogênio e nitrogênio foram determinados em um aparelho PE 2400 CHN Elemental Analyzer. Para determinação do teor de cinzas (minerais) foi utilizada a Norma M 11/77 (ABTCP). ${ }^{11}$

\section{Processo de ativação}

O pergaminho foi impregnado com $\mathrm{ZnCl}_{2}$ na proporção de $1: 1$ $(\mathrm{m} / \mathrm{m})$, seco a $100{ }^{\circ} \mathrm{C}$ por $24 \mathrm{~h}$ e ativado a $500{ }^{\circ} \mathrm{C}$ por $3 \mathrm{~h}$. A ativação foi feita em atmosfera inerte $\left(\mathrm{N}_{2}\right)$ em um forno tubular horizontal (Figura 1). O carvão obtido foi lavado sucessivas vezes com $\mathrm{HCl} /$ $\mathrm{H}_{2} \mathrm{O}$ para a retirada dos resíduos do agente ativante e desobstrução dos poros formados.

\section{Caracterização do carvão ativado do pergaminho (CAP)}

A caracterização do CAP foi realizada a partir das isotermas de adsorção física de $\mathrm{N}_{2}$ (77 K), em um aparelho Quantachrome, modelo NOVA-1200. A área superficial específica foi calculada pelo método BET e as porosidades específicas pelo método $\mathrm{BJH} .{ }^{12} \mathrm{~A}$ superfície do CAP foi analisada por infravermelho com transformada de Fourier (FTIR) em um aparelho Digilab Excalibur-FT3000 e microscopia eletrônica de varredura (MEV) utilizando um aparelho LEO EVO 40XVP. 


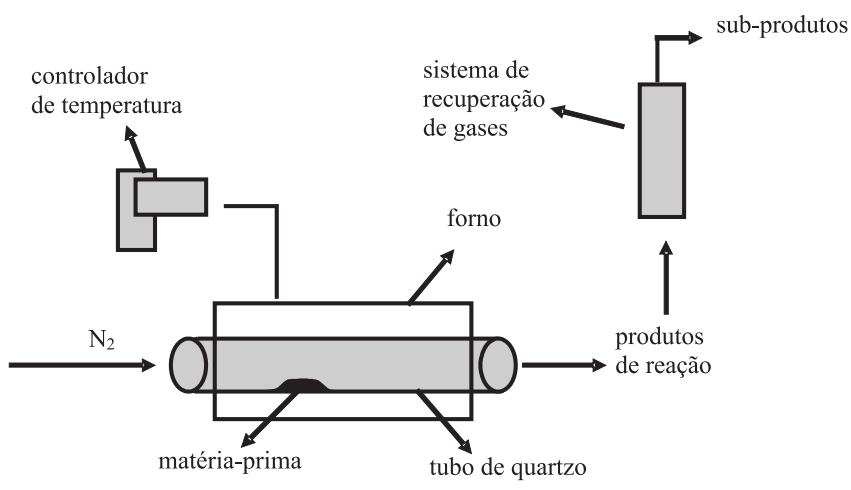

Figura 1. Esquema do forno utilizado na preparação do carvão ativado de pergaminho $(C A P)$

\section{Teste de adsorção}

Para os ensaios de adsorção na fase líquida foi utilizado como adsorbato o corante azul de metileno (AM) da Merck. Para os testes, $10 \mathrm{mg}$ de CAP foram colocados em contato com $10 \mathrm{~mL}$ de soluções de $\mathrm{AM}$ em várias concentrações $(10,25,50,100,250,500$ e 1000 $\mathrm{mg} \mathrm{L}^{-1}$ ) por $24 \mathrm{~h}$ à temperatura ambiente (aproximadamente $25^{\circ} \mathrm{C}$ ) $\mathrm{e}$ pH 5,3. A concentração restante do AM foi analisada em um espectrofotômetro de UV/VIS (Biosystems SP-2000) no comprimento de onda de $645 \mathrm{~nm}$. O mesmo procedimento foi feito com o carvão ativado comercial da marca Merck (CAC) para fim de comparação.

Para determinar a capacidade máxima de adsorção de AM em CAP, a isoterma de adsorção foi conduzida analisando os dados através do modelo de Langmuir pelas Equações 1 e 2:13

$q e q=\frac{K_{L} \cdot C e q \cdot b}{1+K_{L} \cdot C e q}$

Após linearização:

$$
\frac{C e q}{q e q}=\frac{1}{K_{L} \cdot b}+\frac{C e q}{b}
$$

onde qeq $\left(\mathrm{mg} \mathrm{g}^{-1}\right)$ é a quantidade adsorvida de AM por grama de CAP; Ceq $\left(\mathrm{mg} \mathrm{L}^{-1}\right)$ é a concentração de AM no equilíbrio; b (mg g $\left.{ }^{-1}\right)$ é a capacidade de adsorção máxima e $\mathrm{K}_{\mathrm{L}}\left(\mathrm{L} \mathrm{mol}^{-1}\right)$ é uma constante relacionada à energia de adsorção, ambos ( $\left(\mathrm{b}\right.$ e $\mathrm{K}_{\mathrm{L}}$ ) podem ser determinados através do gráfico de Ceq/qeq versus Ceq.

\section{Estudo da cinética de adsorção de AM em CAP}

O estudo foi feito para determinar o tempo necessário para se atingir o equilíbrio de adsorção. Neste experimento $10 \mathrm{mg}$ de CAP juntamente com $10 \mathrm{~mL}$ de solução de AM 10, 50, 100, 250 ou 500 $\mathrm{mg} \mathrm{L}^{-1}$ foram mantidos em contato por diferentes tempos $(0,5 ; 1,0$; 2,$0 ; 4,0 ; 6,0 ; 12$ e 24,0 h) e a concentração de AM restante, após a adsorção, foi determinada por espectrofotometria UV/VIS no comprimento de onda de $645 \mathrm{~nm}$.

\section{Efeito da temperatura na adsorção de AM em CAP e estudos termodinâmicos}

$10 \mathrm{mg}$ de CAP foram mantidas em equilíbrio com $10 \mathrm{~mL}$ de solução de AM em diferentes concentrações (10, 25, 50, 100, 250, 500 e $1000 \mathrm{mg} \mathrm{L}^{-1}$ ). As temperaturas examinadas foram 10, 30, 40 e $60{ }^{\circ} \mathrm{C}$. Após $16 \mathrm{~h}$ de contato a concentração de AM foi determinada por espectrofotometria de UV-VIS.
A energia livre de Gibbs $\left(\Delta \mathrm{G}^{\circ}\right)$, entalpia $\left(\Delta \mathrm{H}^{\circ}\right)$ e entropia $\left(\Delta \mathrm{S}^{\circ}\right)$ foram calculadas usando as Equação 3 e 4 :

$\Delta \mathrm{G}^{\circ}=-\mathrm{RT} \ln \mathrm{K}_{\mathrm{L}}$

em que $\mathrm{T}$ é a temperatura absoluta em Kelvin (K); R é a constante universal dos gases $\left(8,314 \mathrm{~J} \mathrm{~mol}^{-1} \mathrm{~K}^{-1}\right)$ e $\mathrm{K}_{\mathrm{L}}\left(\mathrm{L} \mathrm{mol}^{-1}\right)$ é a constante de adsorção de Langmuir, derivada das isotermas a 10, 30, 40 e $60{ }^{\circ} \mathrm{C}$. De acordo com a Equação de van't Hoff,

$\ln K_{L}=\frac{\Delta S^{o}}{R}-\frac{\Delta H^{o}}{R T}$

$\Delta \mathrm{H}^{\circ}$ e $\Delta \mathrm{S}^{\circ}$ podem ser obtidas, respectivamente, da inclinação e do intercepto de um gráfico de $\ln \mathrm{K}_{\mathrm{L}}$ versus $1 / \mathrm{T}$.

\section{RESULTADOS E DISCUSSÃO}

\section{Caracterização do pergaminho}

A composição do pergaminho é mostrada na Tabela 1 . O baixo teor de cinzas $(1,37 \%)$ no pergaminho é um fator positivo para produção de CAP, visto que a matéria mineral, devido ao caráter hidrofílico, promove a adsorção de água, competindo com outros compostos de interesse. Pode-se observar o aumento no teor de carbono (entre pergaminho e CAP), devido à eliminação de hidrogênio e de oxigênio no processo de pirólise. ${ }^{14}$

Tabela 1. Composição do pergaminho e do carvão ativado de pergaminho (CAP) baseado nas porcentagens de matéria seca (MS)

\begin{tabular}{lccccc}
\hline Material & $\mathrm{C}$ & $\mathrm{H}$ & $\mathrm{N}$ & $\mathrm{O}^{1}$ & Cinzas \\
$(\%)$ & $(\%)$ & $(\%)$ & $(\%)$ & $(\%)$ \\
\hline Pergaminho & 44,00 & 5,41 & 0,67 & 49,92 & 1,37 \\
CAP & 62,30 & 3,36 & 0,67 & 33,67 & -
\end{tabular}

1. O teor de oxigênio foi obtido por diferença

\section{Espectroscopia na região do infravermelho}

Comparando os espectros de FTIR do pergaminho e do CAP (Figura 2a e b, respectivamente) é possível observar a diminuição da intensidade da banda em aproximadamente $3400 \mathrm{~cm}^{-1}$, característica de estiramento vibracional de grupos $\mathrm{OH}$ que, após a formação do CAP, passam a estar, se presentes, preferencialmente nas bordas das camadas grafênicas. ${ }^{14} \mathrm{O}$ desaparecimento da banda em aproximadamente $2929 \mathrm{~cm}^{-1}$, atribuída aos estiramentos vibracionais simétricos e assimétricos de grupos $\mathrm{CH}$, evidencia a ocorrência da pirólise do pergaminho.

\section{Microscopia eletrônica de varredura}

A Figura 3 apresenta as micrografias do pergaminho, CAP e CAC (carvão ativado comercial).

Comparando as micrografias do pergaminho e do CAP, podese observar a modificação da estrutura do precursor após o processo de pirólise e o aparecimento de uma superfície irregular e porosa, que se assemelha com a do CAC.

\section{Área BET e porosidade}

A forma da isoterma de adsorção/dessorção de nitrogênio a 77 $\mathrm{K}$ para o CAP pode fornecer informações qualitativas preliminares 


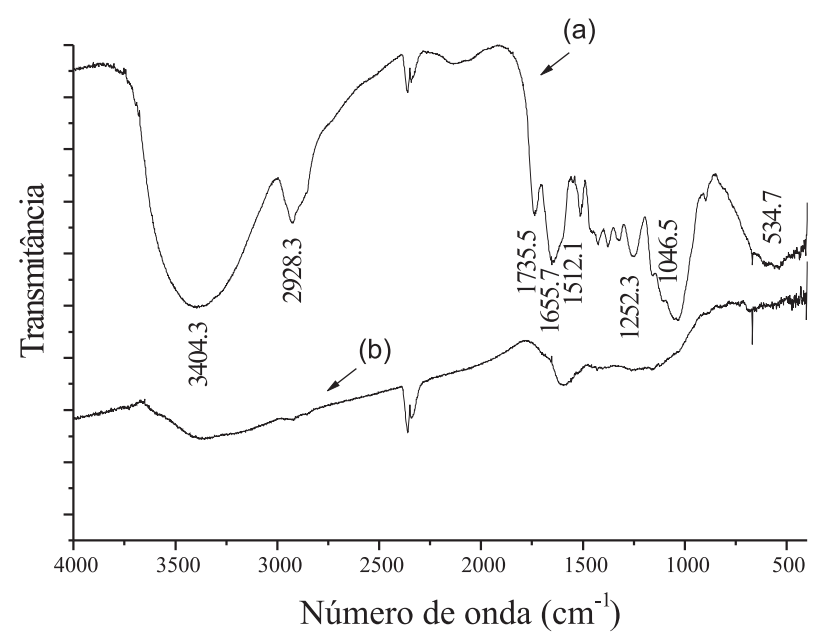

Figura 2. Espectros de FTIR do pergaminho (a) e do carvão ativado de pergaminho - CAP (b)

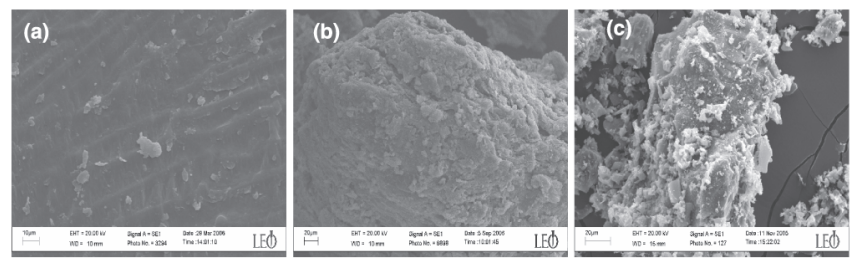

Figura 3. Micrografias (a) pergaminho (b) carvão ativado de pergaminho CAP e (c) carvão ativado comercial - CAC

sobre o mecanismo de adsorção e da estrutura porosa do carvão. Através da classificação de isotermas proposta por BET, observouse que a isoterma obtida é do tipo I, típica de sólidos microporosos. ${ }^{12}$

A confirmação de que a estrutura do CAP é constituída, na maior parte, por microporos é mostrada na Figura 4. A área total obtida por BET foi de 521,6 $\mathrm{m}^{2} \mathrm{~g}^{-1}$.

Pela distribuição de diâmetro de poro do CAP pode-se observar que a grande maioria dos poros possui tamanho inferior a $20 \AA$. A área superficial de microporos foi de $377,2 \mathrm{~m}^{2} \mathrm{~g}^{-1}$, ou seja, mais de $70 \%$ da área total do CAP. Os CAs são geralmente microporosos, proporcionando alta capacidade de adsorção de moléculas de dimensões pequenas, tais como gases e diversos solventes. Porém devem também ter macro e mesoporos, importantes para a adsorção de moléculas grandes, além de servirem como meio de transporte. A área superficial mesoporosa do CAP foi encontrada pela diferença da área superficial total e de microporos, sendo de $144,3 \mathrm{~m}^{2} \mathrm{~g}^{-1}$.

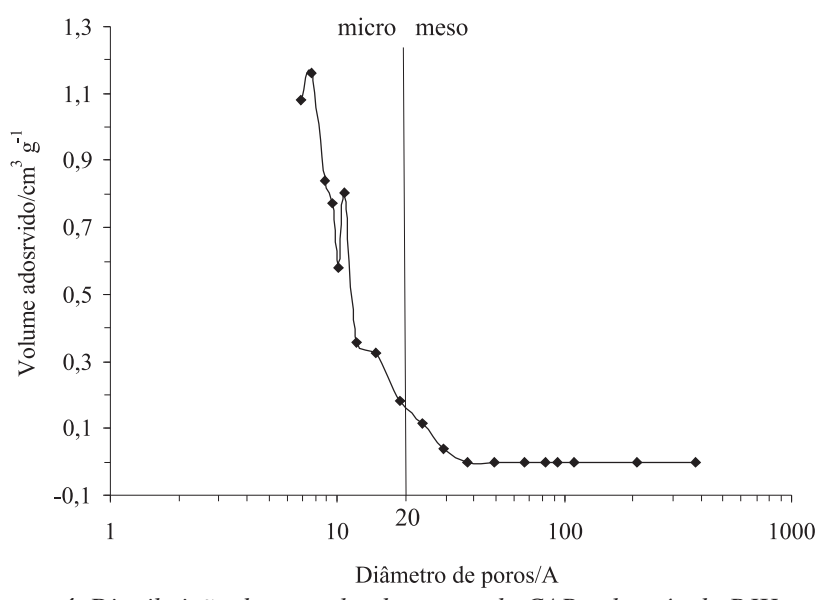

Figura 4. Distribuição do tamanho dos poros do CAP pelo método BJH

\section{Adsorção do azul de metileno}

A adsorção na fase líquida aplica-se a muitos processos de purificação, sendo o principal tratamento de resíduos em água. O AM é, freqüentemente empregado como adsorbato na adsorção em fase líquida. As isotermas de adsorção de AM em CAP e CAC são mostradas na Figura 5, que relaciona quantidade adsorvida de AM por massa do CAP e CAC (qeq) e as concentrações restantes de AM (Ceq) nas soluções aquosas.

As isotermas obtidas podem ser classificadas, de acordo com a IUPAC, como do tipo I, freqüentemente chamadas do tipo Langmuir. Esse tipo de isoterma indica que adsorbato e adsorvente têm alta afinidade. Deste modo, o modelo de adsorção de Langmuir foi adotado para calcular a capacidade máxima de adsorção de AM. O CAP apresentou adsorção máxima de aproximadamente $188,7 \mathrm{mg} \mathrm{g}^{-1}$, um valor satisfatório se comparado com o carvão ativado comercial (232,6 $\left.\mathrm{mg} \mathrm{g}^{-1}\right)$ que tem área superficial maior $\left(933,0 \mathrm{~m}^{2} \mathrm{~g}^{-1}\right)$.

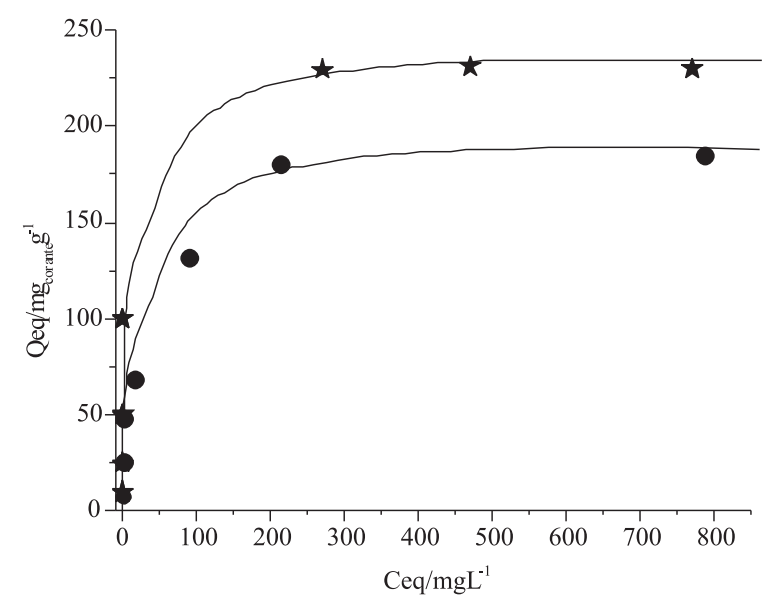

Figura 5. Isotermas de adsorção de AM em CAP (•) e em CAC ( $\star$ ). $10 \mathrm{mg}$ de carvão em $10 \mathrm{~mL}$ de solução de AM a 10, 25, 50 100, 250, 500 e $1000 \mathrm{mg} \mathrm{L}^{-1}$, $24 h, 25^{\circ} \mathrm{C}$

Os dados apresentados na Tabela 2 mostram que, apesar do $\mathrm{CAP}$ apresentar área superficial $\left(\mathrm{S}_{\mathrm{BET}}\right)$ e adsorção máxima (b) menores que o carvão comercial, ele adsorveu maior quantidade de corante por metro quadrado.

Tabela 2. Valores de área BET $\left(\mathrm{S}_{\mathrm{BET}}\right)$ e adsorção máxima (b) de azul de metileno (AM) em CAP e em CAC

\begin{tabular}{lccc}
\hline & $\begin{array}{c}\mathrm{S}_{\mathrm{BET}}{ }^{1} \\
\left(\mathrm{~m}^{2} \mathrm{~g}^{-1}\right)\end{array}$ & $\begin{array}{c}\mathrm{b}^{2} \\
\left(\mathrm{mg} \mathrm{g}^{-1}\right)\end{array}$ & $\begin{array}{c}\mathrm{AMAd}^{3} \\
\left(\mathrm{mg} \mathrm{m}^{-2}\right)\end{array}$ \\
\hline $\mathrm{CAC}^{4}$ & 933,0 & 232,6 & 0,25 \\
$\mathrm{CAP}^{5}$ & 521,5 & 188,7 & 0,36 \\
\hline
\end{tabular}

1. área BET; 2. adsorção máxima de azul de metileno no carvão, 3. mg de azul de metileno adsorvido por metro quadrado de carvão; 4. carvão ativado comercial, 5. carvão ativado de pergaminho.

\section{Efeito do tempo sobre adsorção do AM em CAP}

A capacidade de adsorção de AM pelo CAP é função do tempo de contato entre adsorvente e adsorbato e da concentração do adsorbato. A Figura 6 mostra uma rápida diminuição da concentração do AM na solução nas primeiras horas. Após 14 h de contato, a remoção de AM pelo CAP é superior a 97\% quando a concentração inicial de adsorbato 


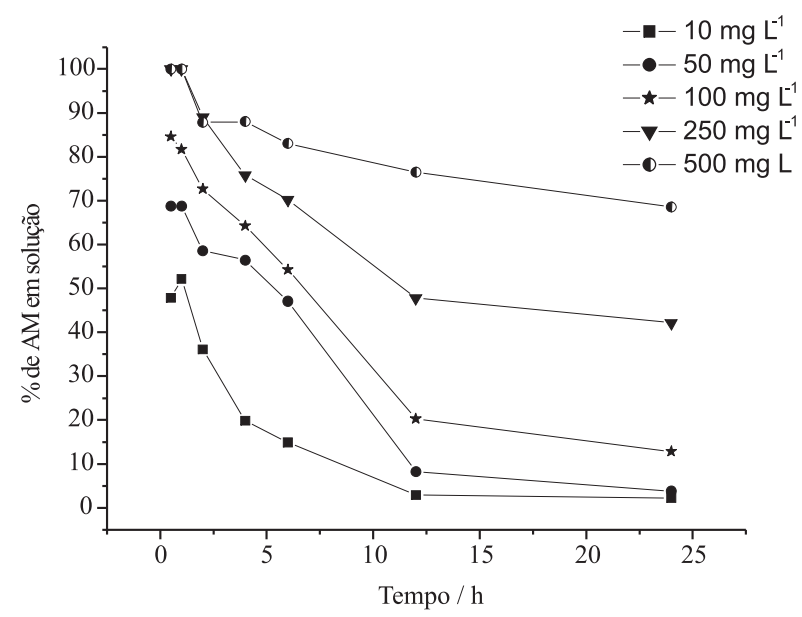

Figura 6. Efeito do tempo $(0,5 ; 1 ; 2 ; 4 ; 6 ; 12$ e 24 h) na adsorção de AM(10, 50, 100, 250 ou $\left.500 \mathrm{mg} \mathrm{L}^{-1}\right) \mathrm{em} \mathrm{CAP}\left(25^{\circ} \mathrm{C}\right)$

é de $10 \mathrm{mg} \mathrm{L}^{-1}$ e de $23 \%$ quando a concentração de $\mathrm{AM}$ é de $500 \mathrm{mg}$ $\mathrm{L}^{-1}$. A partir deste tempo, tem-se um equilíbrio, ou seja, a quantidade adsorvida de AM não se altera significativamente.

A área superficial do CAP, estimada a partir da adsorção de $\mathrm{AM}\left(\mathrm{S}_{\mathrm{AM}}\right)$, foi obtida pela Equação $5^{9}$

$\mathrm{S}_{\mathrm{AM}}=\mathrm{S}_{\mathrm{AM}}^{\circ} \cdot \mathrm{b}$

onde $\mathrm{S}_{\mathrm{AM}}{ }^{\circ}$ é a área superficial do $\mathrm{AM}\left(1,93 \mathrm{~m}^{2} \mathrm{mg}^{-1}\right)$ e b é a capacidade máxima de adsorção de $\mathrm{AM}$ em $\operatorname{CAP}\left(188,7 \mathrm{mg} \mathrm{g}^{-1}\right)$.

Os parâmetros para as isotermas de adsorção e a área superficial, $\mathrm{S}_{\mathrm{AM}}$, são mostrados na Tabela 3 .

A S $_{\mathrm{AM}}$ obtida foi de $364,2 \mathrm{~m}^{2} \mathrm{~g}^{-1}$, valor muito próximo ao da área de microporos $\left(\mathrm{S}_{\text {microporos }}\right)$. Este resultado pode estar relacionado ao fato de que os microporos apresentam diâmetros de poro que variam de 0,2-2,0 $\mathrm{nm}$ e, segundo Barton, ${ }^{15}$ a molécula do corante catiônico, AM, tem uma secção transversal molecular de aproximadamente $0,8 \mathrm{~nm}$. Estima-se que o diâmetro mínimo dos poros de um adsorvente na qual esta molécula é acessível seja de 1,3 nm, mas este resultado não significa que o $\mathrm{AM}$ tenha sido adsorvido somente pelos microporos, mas também pelos mesoporos.

A relação $S_{A M} / S_{\mathrm{BET}}$ é uma indicação da fração da porosidade acessível ao AM. Como pode ser visto na Tabela 3, as moléculas do corante ocupam aproximadamente $70 \%$ da porosidade total encontrada pelo BET.

Tabela 3. Características de adsorção de AM em CAP

\begin{tabular}{lcccccc}
\hline & $\begin{array}{c}\mathrm{S}_{\mathrm{BET}}{ }^{1} \\
\left(\mathrm{~m}^{2} \mathrm{~g}^{-1}\right)\end{array}$ & $\begin{array}{c}\mathrm{b}^{2} \\
\left(\mathrm{mg} \mathrm{g}^{-1}\right)\end{array}$ & $\begin{array}{c}\mathrm{S}_{\text {microporos }}{ }^{3} \\
\left(\mathrm{~m}^{2} \mathrm{~g}^{-1}\right)\end{array}$ & $\begin{array}{c}\mathrm{S}_{\text {mesoporos }}{ }^{4} \\
\left(\mathrm{~m}^{2} \mathrm{~g}^{-1}\right)\end{array}$ & $\begin{array}{c}\mathrm{S}_{\mathrm{AM}}{ }^{5} \\
\left(\mathrm{~m}^{2} \mathrm{~g}^{-1}\right)\end{array}$ & $\mathrm{S}_{\mathrm{AM}} / \mathrm{S}_{\text {BET }}$ \\
\hline CAP & 521,5 & 188,7 & 377,2 & 144,3 & 364,2 & 0,70 \\
\hline
\end{tabular}

1. área BET; 2. adsorção máxima de azul de metileno no carvão ativado de pergaminho, 3 . área de microporos; 4. área de mesoporos, 5. área superficial estimada a partir da adsorção de azul de metileno.

\section{Parâmetros termodinâmicos}

Os valores de $\Delta \mathrm{G}^{\circ}$ foram obtidos a partir das constantes de Langmuir na várias temperaturas $\left(10,30,40\right.$ e $\left.60{ }^{\circ} \mathrm{C}\right)$. Os valores de $\Delta \mathrm{H}^{\circ}$ e $\Delta \mathrm{S}^{\circ}$ foram obtidos da inclinação e do intercepto da reta $\ln \mathrm{K}_{\mathrm{L}}$ x 1/T, representada na Figura 7. Os resultados para os parâmetros termodinâmicos da adsorção de AM em CAP são listados na Tabela 4. Observa-se que os valores de $\Delta \mathrm{G}^{\circ}$ são negativos, indicando que o

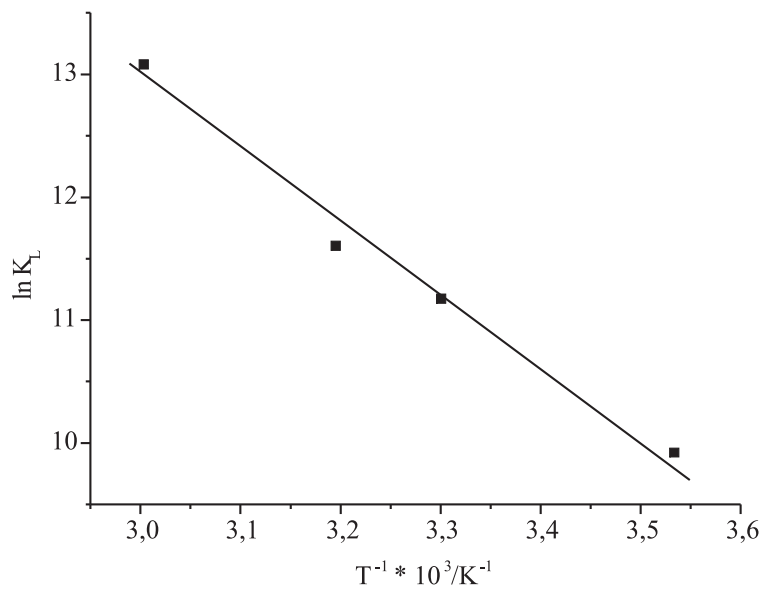

Figura 7. Variação no equilíbrio de adsorção do AM pelo CAP com a temperatura $\left(10,30,40\right.$ e $\left.60^{\circ} \mathrm{C}\right)$

processo de adsorção de AM em CAP é espontâneo. $\mathrm{O}$ aumento da temperatura de 10 para $60{ }^{\circ} \mathrm{C}$ provocou a diminuição de $\Delta \mathrm{G}^{\circ}$ de $-23,3$ para $-36,2 \mathrm{~kJ} \mathrm{~mol}^{-1}$, ou seja, favoreceu o processo de adsorção e, conseqüentemente, aumentou a capacidade adsortiva do CAP.

$\mathrm{O}$ sinal positivo de $\Delta \mathrm{H}^{\circ}$ mostra que a adsorção de $\mathrm{AM}$ em CAP é um processo endotérmico. $\mathrm{O}$ valor positivo de $\Delta \mathrm{S}^{\circ}\left(253,73 \mathrm{~J} \mathrm{~mol}^{-1} \mathrm{~K}^{-1}\right)$ indica uma alta tendência à desordem na interface CAP-Solução AM.

Tabela 4. Parâmetros termodinâmicos calculados a partir de dados das isotermas de adsorção de Langmuir

\begin{tabular}{lcccccr}
\hline $\begin{array}{l}\mathrm{T} \\
\left({ }^{\circ} \mathrm{C}\right)\end{array}$ & $\begin{array}{c}\mathrm{b}^{1} \\
\left(\mathrm{mg} \mathrm{g}^{-1}\right)\end{array}$ & $\begin{array}{c}\mathrm{K}_{\mathrm{L}}^{2} \\
\left(\mathrm{~L} \mathrm{~mol}^{-1}\right)\end{array}$ & \multicolumn{5}{c}{$\left.\begin{array}{c}\Delta \mathrm{G}^{\circ} \\
\left(\mathrm{kJ} \mathrm{mol}^{-1}\right)\end{array}\right) \begin{array}{c}\Delta \mathrm{H}^{\circ} \\
\left(\mathrm{kJ} \mathrm{mol}^{-1}\right)\left(\mathrm{J} \mathrm{mol}^{-1} \mathrm{~K}^{-1}\right)\end{array}$} \\
\hline 10 & 105,26 & 20327,94 & 0,9973 & $-23,34$ & & \\
30 & 126,58 & 71188,49 & 0,9945 & $-28,15$ & 48,67 & 253,73 \\
40 & 156,25 & 109483,68 & 0,9994 & $-30,20$ & & \\
60 & 196,08 & 479846,45 & 0,9997 & $-36,22$ & & \\
\hline
\end{tabular}

1. adsorção máxima de azul de metileno no carvão ativado de pergaminho, 2. constante de adsorção de Langmuir; 3. coeficiente de correlação.

\section{CONCLUSÕES}

O pergaminho mostrou-se um bom precursor para a produção de carvão ativado. Sua capacidade máxima de adsorção para a molécula de AM (188 $\left.\mathrm{mg} \mathrm{g}^{-1}\right)$ é comparável à do carvão ativado comercial (232,6 $\mathrm{mg} \mathrm{g}^{-1}$ ). Independente da concentração de adsorbato em solução (10, 50, 100, $250 \mathrm{ou} 500 \mathrm{mg} \mathrm{L}^{-1}$ ), após um período de aproximadamente 14 $\mathrm{h}$ não ocorre mais adsorção de $\mathrm{AM}$, o sistema entra em equilíbrio e a quantidade adsorvida não se altera com o tempo. A capacidade de adsorção de AM pelo CAP sofre um aumento considerado (de 105 para $196 \mathrm{mg} \mathrm{g}^{-1}$ ) com o aumento da temperatura $\left(10\right.$ para $\left.60{ }^{\circ} \mathrm{C}\right)$, mostrando a natureza endotérmica das reações. $\mathrm{O}$ que é comprovado pelo valor positivo de $\Delta \mathrm{H}^{\circ}$ obtido $\left(48,67 \mathrm{~kJ} \mathrm{~mol}^{-1}\right)$. As interações são termodinamicamente estáveis, como sugerem os valores negativos da energia livre de Gibbs.

\section{AGRADECIMENTOS}

Ao CNPq pela concessão da bolsa de mestrado. À Central de Análise e Prospecção Química da Universidade Federal de Lavras (CAPQ/UFLA), onde foi possível a realização das análises. À EPAMIG pelo fornecimento do pergaminho. 


\section{REFERÊNCIAS}

1. http://www.conab.gov.br/conabweb/download/safra/1_levantamento_2008.pdf, acessada em Junho 2008.

2. Venturim, J. B.; Tese de Doutorado, Universidade Federal de Santa Catarina, Brasil, 2002.

3. Brum, S. S.; Dissertação de Mestrado, Universidade Federal de Lavras, Brasil, 2007.

4. Lussier, M. G.; Shull, J. C.; Miller, D. J.; Carbon 1994, 32, 1493.

5. Castro, J. B.; Bonelli, P. R.; Cerrella, E. G.; Cukierman, A. L.; Ind. Eng. Chem. Res. 2000, 39, 4166.

6. Yilmaz, O.; Kantarli, I. C.; Yuksel, M.; Saglam, M.; Yanik, J.; Resources Conservation and Recycling 2007, 49, 436.

7. Skodras, G.; Diamantopouiou, I.; Zabaniotou, A.; Stavropoulos, G.; Sakellaropoulos, G. P.; Fuel Process. Technol. 2007, 88, 749.
8. Reinoso, R. F.; Marsh, H.; Activated Carbon, $1^{\text {st }}$ ed., Elsevier: London, 2006.

9. Stavropoulos, G. G.; Zabaniotou, A. A.; Microporous Mesoporous Mater. 2005, 82, 79.

10. Almansa, C.; Rodriguez-Reinoso, F.; Molina-Sabio, M.; Microporous Mesoporous Mater. 2004, 76, 185

11. Associação Brasileira Técnica de Celulose e Papel-ABTCP; Normas técnicas ABCP, ABTCP: São Paulo, 1974.

12. Gregg, S. J.; Sing, K. S. W. H.; Adsorpition, Superface, Area and Porosity, $2^{\text {nd }}$ ed., Academic Press: New York, 1982.

13. Giles, C. H.; MacEwan, T. H.; Nakhwa, S. N.; Smith, D.; J. Chem. Soc. 1960, 3973 .

14. Castilla, M. C. Em Adsorbentes en la solución de algunos problemas ambientales; Reinoso, F. R.; ed.; Universidade de Granada: Espanha, 2004, cap. 4.

15. Barton, S. S.; Carbon 1987, 25, 343. 